Pacific Journal of Mathematics

CHARACTERIZING GLOBAL PROPERTIES IN INVERSE (z) 


\title{
CHARACTERIZING GLOBAL PROPERTIES IN INVERSE LIMITS
}

\author{
ZVONKO ČERIN
}

To Georgle

\begin{abstract}
This paper presents necessary and sufficient conditions, in terms of properties of bonding maps and bonding spaces, on an inverse sequence $\underline{X}=\left\{X_{l}, p_{t+1}\right\}$ of compact metric spaces in order that its inverse limit $\bar{X}=\lim \underline{X}$ is either an approximate absolute neighborhood retract, an (internally) $e$-calm compactum, an absolute neighborhood retract, an $L C^{n}$ compactum, or that $X$ has (covering) dimension $\leq n$.
\end{abstract}

1. Introduction. Let $X$ denote the inverse limit of an inverse sequence $\underline{X}=\left\{X_{l}, p_{i+1}\right\}$ of compact metric spaces. The main purpose of this paper is to identify necessary and sufficient conditions which will insure that $X$ is either an approximate absolute neighborhood retract (both in the sense of Clapp [Cl] $\left(\mathrm{AANR}_{\mathrm{C}}\right)$ and in the sense of Noguchi [No] $\left(\mathrm{AANR}_{\mathrm{N}}\right)$ ), an (internally) e-calm compactum [Č́1], an absolute neighborhood retract (ANR), an $L C^{n}$ compactum, or that $X$ has dimension $\leq n$.

The problem of characterizing the dimension of the inverse limit of an inverse system was studied earlier by Pasynkov [Pa] and by Delinic and Mardešić [DM]. On the other hand, Fort and Segal [FS, Theorems 2 and 3] considered a surjective inverse sequence $\underline{X}=\left\{X_{i}, p_{i+1}\right\}$ (i.e., an inverse sequence with all bonding maps $p_{i_{\imath+1}}$ onto) of locally connected continua and discovered that each bonding space $X_{i}$ can be embeddded as a subset $X_{l}^{*}$ of the product $P=\prod_{l>0} X_{l}$ (see $\S 3$ ) in such a way that the inverse limit $X$ (considered as a subset of $P$ ) is a locally connected continuum iff the sequence $X_{1}^{*}, X_{2}^{*}, \ldots$ converges 0 -regularly to $X[\mathbf{W h}$. Another characterization of local connectedness in inverse limits was given by Gordh and Mardešić [GM]. They introduced a notion of local connectedness for inverse systems and proved that the inverse limit $X$ of a surjective inverse system $\underline{X}=\left\{X_{\alpha}, p_{\alpha \alpha^{\prime}}, A\right\}$ of locally connected continua is locally connected iff $\underline{X}$ is locally connected.

Our approach is motivated by shape theory and represents an applica-

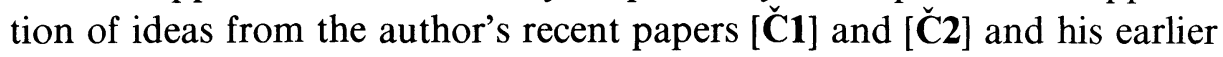




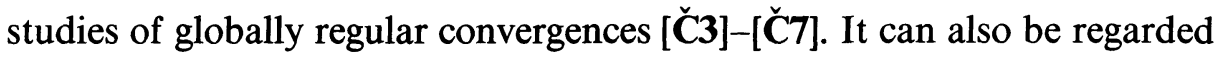
as a natural extension of techniques both from [FS] and [GM].

The following is a brief description of our method for the case of AANR $_{C}$ 's.

First we observe that the Mardešić-Segal treatment of movability in [MS2] and the author's notion of movably regular convergence [Č3] provide the following characterization.

(1.1) For an inverse ANR-sequence $\underline{X}=\left\{X_{i}, p_{i i+1}\right\}$ the following are equivalent:

(i) $X=\lim \underline{X}$ is movable [B].

(ii) $X$ is movable [MS2].

(iii) The sequence $X_{1}^{*}, X_{2}^{*}, \ldots$ converges movably regularly to $X$ [亡̌3].

Then we use Corollary (4.3) in [Č3] which shows that AANR ${ }_{C}$ 's agree with $e$-movable compacta and perform changes necessary to make

$e-(1.1):$ For a surjective ANR-sequence $\underline{X}=\left\{X_{i}, p_{i+1}\right\}$ the following are equivalent:

(i) $X=\lim \underline{X}$ is $e$-movable.

(ii) $\underline{X}$ is $e$-movable.

(iii) The sequence $X_{1}^{*}, X_{2}^{*}, \ldots$ converges $e$-movably regularly to $X$ [ک̌6].

a true statement (see Theorem (4.2)). This requires defining a notion of $e$-movability for inverse sequences which is straightforward if one recalls that (roughly speaking) the concept of an $e$-movable compactum is obtained from Borsuk's original concept of a movable compactum by replacing homotopies with $\varepsilon$-homotopies.

In order to get characterizations of ANR's, $L C^{n}$ compacta, (internally) $e$-calm compacta, and dimension, we shall "rigidify" (using results

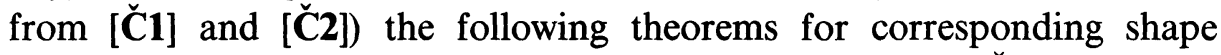
invariants of strong movability [B], strong $n$-movability [Č8], calmness [Č9], and fundamental dimension [B], respectively.

(1.2) Let $\underline{X}=\left\{X_{i}, p_{i+1}\right\}$ be an inverse ANR-sequence. The following are equivalent.

(i) $X=\lim \underline{X}$ is strongly movable (strongly $n$-movable).

(ii) $\underline{X}$ is strongly movable [M1] (strongly $n$-movable).

(iii) The sequence $X_{1}^{*}, X_{2}^{*}, \ldots$ converges strongly movably regularly (strongly $n$-movably regularly) to $X$.

The strongly movably regular convergence in (iii) is less restrictive than the weakly $\mathscr{P}_{p}$-movably regular convergence in [Č5] and is defined as follows. A sequence $\left\{A_{i}\right\}_{i=1}^{\infty}$ of compacta in a metric space $Y$ converges strongly movably regulary (strongly n-movably regularly) to a compactum 
$A_{0}, A_{0} \subset Y$, provided in some, and hence in every, ANR $M$ which contains $Y$, for every neighborhood $U$ of $A_{0}$ in $M$ there is a neighborhood $V$ of $A_{0}$ in $M, V \subset U$, such that for every neighborhood $W$ of $A_{0}$ in $M$ there is an index $i_{W}$ with the property that for every $i \geq i_{W}$ there is a neighborhood $W_{0}^{i}$ of $A_{i}$ in $M, W_{0}^{i} \subset V \cap W$, so that for every $\mathscr{P}_{p}$-map (for every $\mathscr{P}_{p}^{n}$-map) (see §2) $f:\left(K, K_{0}\right) \rightarrow\left(V, W_{0}^{i}\right)$ there is a homotopy $f_{t}$ : $K \rightarrow U, 0 \leq t \leq 1$, with $f_{0}=f, f_{1}(K) \subset W$, and $f_{1}\left|K_{0}=f\right| K_{0}$.

(1.3) For an inverse ANR-sequence $\underline{X}=\left\{X_{i}, p_{i+1}\right\}$ the following are equivalent.

(i) $X=\lim \underline{X}$ is calm.

(ii) $\underline{X}$ is calm (i.e. $\underline{X}$ satisfies (4.2)(vi) in [Č 9]).

(iii) The sequence $X_{1}^{*}, X_{2}^{*}, \ldots$ converges calmly regularly to $X$.

The calmly regular convergence is weaker than $\mathcal{P}$-calmly regular convergence studied in [ČC]. Its definition is analogous to the above definition of the strongly movably regular convergence (see (5.5)).

(1.4) (Nowak [N] and Čerin [Č10]) The inverse limit $X$ of an inverse ANR-sequence $\underline{X}=\left\{X_{i}, p_{i+1}\right\}$ has fundamental dimension $\leq n$ iff $\underline{X}$ is $n$-tame (i.e., iff for every index $i$ there is $j \geq i$, an at most $n$-dimensional finite polyhedron $P$, and maps $\alpha: X_{j} \rightarrow P$ and $\beta: P \rightarrow X_{i}$ such that the diagram

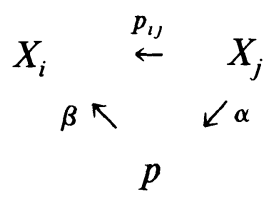

is homotopy commutative).

We thank the referee for helpful suggestions (especially for Remark $(6.4))$.

2. Preliminaries. Throughout the paper $\mathscr{P}$ will denote the class of all compact ANR's and $\mathscr{P}_{p}$ will denote the class of all pairs $\left(K, K_{0}\right)$ where $K$ and $K_{0}$ are compact ANR's and $K_{0}$ is a subset of $K$. By $\mathscr{P}^{n}\left(\mathscr{P}_{p}^{n}\right)$ we denote all $K \in \mathscr{P}\left(\left(K, K_{0}\right) \in \mathscr{P}_{p}\right)$ with $\operatorname{dim} K \leq n$.

A map $f: K \rightarrow Y$ is called a $\mathscr{P}$-map provided $K \in \mathscr{P}$. Similarly, a map of pairs $f:\left(K, K_{0}\right) \rightarrow\left(Y, Y_{0}\right)$ is a $\mathscr{P}_{p}$-map if $\left(K, K_{0}\right) \in \mathscr{P}_{p}$.

We shall say that maps $f$ and $g$ of a space $Z$ into a metric space $(Y, d)$ are $\varepsilon$-close provided $d(f(z), g(z))<\varepsilon$ for every $z \in Z$. If $Z$ and $W$ are subsets of $Y$ and the composition of $f: Z \rightarrow W$ with the inclusion of $W$ into $Y$ is $\varepsilon$-close to the inclusion of $Z$ into $Y$, we call $f$ an $\varepsilon$-map. 
Two maps $f, g: Z \rightarrow Y$ of a space $Z$ into a metric space $(Y, d)$ are $\varepsilon$-homotopic (and we write $f \simeq_{\varepsilon} g$ ) if there is a homotopy $h_{t}: Z \rightarrow Y$, $0 \leq t \leq 1$, between $f$ and $g$ (called an $\varepsilon$-homotopy) such that $h_{0}$ and $h_{t}$ are $\varepsilon$-close for all $t \in I=[0,1]$.

For a metric space $(Y, d), 2^{Y}$ denotes the hyperspace of all nonempty compacta in $Y$ with the Hausdorff metric $d_{\mathrm{H}}$, while $d_{c}$ denotes Borsuk's metric of continuity defined by

$$
d_{c}(A, B)=\inf \{\varepsilon \mid \exists \varepsilon \text {-maps } f: A \rightarrow B \text { and } g: B \rightarrow A\}
$$

for $A, B \in 2^{Y}$. We shall also need the sup-norm metric $d$ on the collection $\operatorname{Map}(Z, Y)$ of all maps of a compact space $Z$ into $Y$ given by

$$
d(f, g)=\sup \{d(f(z), g(z)) \mid z \in Z\}
$$

for $f, g \in \operatorname{Map}(Z, Y)$.

Let $A$ be a subset of a metric space $(Y, d)$, let $U$ and $V, V \subset U$, be open subsets of $Y$ which contain $A$, and let $\varepsilon>0$ and $\delta>0$ be given. Then $\mathcal{P}^{\varepsilon}(U, V ; A), \mathcal{P}_{h}^{\varepsilon}(V, \delta ; A)$, and $\mathcal{P}_{p}^{\varepsilon}(U, V ; A)$ will denote the following statements.

${ }^{\rho} \varepsilon(U, V: A)$ For every neighborhood $W$ of $A$ in $Y$ and every

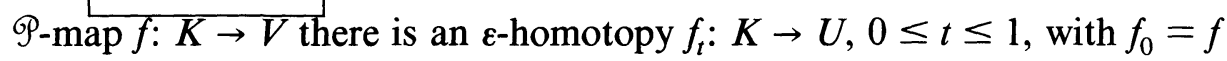
and $f_{1}(K) \subset W$.

$\mathscr{P}_{h}^{\varepsilon}(V, \delta ; A)$ For every neighborhood $W$ of $A$ in $Y$ there is a neighborhood $W_{0}$ of $A$ in $Y, W_{0} \subset V \cap W$, such that every two $\delta$-close

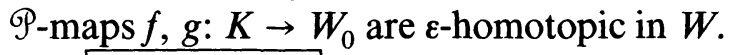

$\mathscr{P}_{p}^{\varepsilon}(U, V: A) \quad$ For every neighborhood $W$ of $A$ in $Y$ there is a neighborhood $W_{0}$ of $A$ in $Y, W_{0} \subset V \cap W$, such that for every $\mathscr{P}_{p}$-map $f$ : $\left(K, K_{0}\right) \rightarrow\left(V, W_{0}\right)$ there is an $\varepsilon$-homotopy $f_{t}: K \rightarrow U, 0 \leq t \leq 1$, with $f_{0}=f, f_{1}(K) \subset W$, and $f_{1}\left|K_{0}=f\right| K_{0}$.

A compactum $A$ is (strongly) e-movable if for some, and hence for every, embedding of $A$ into an ANR $M$ the following holds. For each neighborhood $U$ of $A$ in $M$ and every $\varepsilon>0$ there is a neighborhood $V$ of $A$ in $M, V \subset U$, such that $\left(\mathscr{P}_{p}^{\varepsilon}(U, V ; A)\right) \mathscr{P}^{\varepsilon}(U, V ; A)$ is true. We proved in

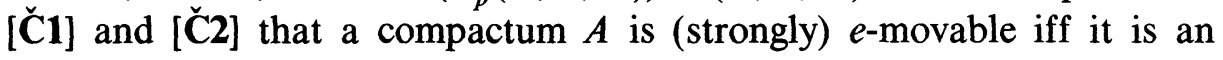
$\mathrm{AANR}_{\mathrm{C}}$ (an ANR).

A compactum $A$ is $e$-calm if for some, and hence for every, embedding of $A$ into an ANR $M$ the following holds. For every $\varepsilon>0$ there is a neighborhood $V$ of $A$ in $M$ and a $\delta>0$ such that $\mathscr{P}_{h}^{\varepsilon}(V, \delta ; A)$ is true. If for every $\varepsilon>0$ there is a $\delta>0$ such that $\delta$-close $\mathscr{P}$-maps into $A$ are $\varepsilon$-homotopic in every neighborhood of $A$ in $M$ then $A$ is internally e-calm. We 
proved in [ $\check{\mathbf{C}} \mathbf{1}]$ that a compactum $A$ is strongly $e$-movable iff $A$ is $e$-movable and (internally) $e$-calm.

For a compact ANR $M$ and an $\varepsilon>0$, let $\Gamma(M, \varepsilon)\left(\Gamma^{*}(M, \varepsilon)\right)$ be the set of all $\delta>0$ such that, for any $\delta$-close maps $f, g: Y \rightarrow M$ defined on a metrizable space $Y$ (and any $\delta$-homotopy $j_{t}: A \rightarrow M, 0 \leq t \leq 1$, defined on a closed subspace $A$ of $Y$ with $j_{0}=f \mid A$ and $j_{1}=g \mid A$ ), there exists an $\varepsilon$-homotopy $h_{t}: Y \rightarrow M, 0 \leq t \leq 1$, such that $h_{0}=f, h_{1}=g$, (and $h_{t} \mid A=$ $j_{t} \mid A$ for every $\left.t \in I\right)([\mathbf{H u}$, p. 122]).

For a map $f: A \rightarrow B$ between metric spaces, let $\Lambda(f, \varepsilon)$ be the set of all $\delta>0$ with the property that $d(x, y)<\delta$ in $A$ implies $d(f(x), f(y))<\varepsilon$ in $B$.

Throughout the paper $\underline{X}=\left\{X_{i}, p_{i+1}\right\}$ will denote an inverse sequence where each $X_{i}$ is a compact metric space and $p_{i+1}: X_{i+1} \rightarrow X_{i}$ is a continuous map. $X=\lim \underline{X}$ will denote the inverse limit of $\underline{X}$, while $p_{i}$ : $X \rightarrow X_{i}$ is a projection. $\stackrel{\leftarrow}{\text { For }} j>i, p_{i j}=p_{i+1} \circ p_{i+1 i+2} \circ \cdots \circ p_{j-1 j}$ and $p_{i i}=\mathrm{id}_{X_{i}}$. If each bonding space $X_{i}$ is an ANR, $\underline{X}$ will be called an inverse ANR-sequence. Inverse $L C^{n}$-sequences and inverse $\mathrm{AANR}_{C}$-sequences are defined analogously.

3. Fort-Segal embeddings. This section describes the method due to Fort and Segal [FS] of embedding the bonding spaces $X_{i}$ of a surjective inverse sequence $\underline{X}=\left\{X_{i}, p_{i+1}\right\}$ into the product $P=\prod_{i>0} X_{i}$ in such a way that the images $X_{l}^{*}$ converge to the inverse limit $X \subset P$. Since we shall study global properties of $X$ in an ANR, we must slightly modify their procedure in order to get that $P$ is nicely embedded in a Hilbert cube.

Let $X_{i}$ be a compactum in a Hilbert cube $Q_{i}$ for each positive integer $i$, let $p_{i+1}$ be a mapping of $X_{i+1}$ onto $X_{i}$, and let $D_{i}$ be a metric for $Q_{i}$ so that $D_{i}(x, y) \leq 1$ for all $x$ and $y$ in $Q_{i}$. Let $\bar{p}_{i+1}: Q_{i+1} \rightarrow Q_{i}$ be an extension of $p_{i+1}$. Let $\left(X, p_{i}\right)=\lim \left\{X_{i}, p_{i+1}\right\}$ and let $\left(\bar{Q}, \bar{p}_{\imath}\right)$ $=\lim \left\{Q_{i}, \bar{p}_{i+1}\right\}$. Note that $X$ is a subset of $\bar{Q}$ and $\bar{p}_{i}$ is an extension of $p_{i}$ for every $i>0$. We then define

$$
d_{i}(x, y)=\sum_{j=1}^{i} 2^{-j} D_{j}\left(\bar{p}_{i j}(x), \bar{p}_{i j}(y)\right)
$$

for each positive integer $i$ and all $x$ and $y$ in $Q_{l}$, and we define

$$
d(u, v)=\sum_{j=1}^{\infty} 2^{-j} D_{j}\left(\bar{p}_{j}(u), \bar{p}_{j}(v)\right)
$$


for all $u$ and $v$ in $\bar{Q}$. Then $d$ is a metric for $\bar{Q}$ and $d_{i}$ is a metric for $Q_{i}$ for each $i>0$. Moreover $\lim _{l \rightarrow \infty} d_{t}\left(\bar{p}_{l}(u), \bar{p}_{i}(v)\right)=d(u, v)$ uniformly on $\bar{Q} \times \bar{Q}$.

Let $Q=\Pi_{i>0} Q_{i}$. If we define a metric $d^{*}$ for $Q$ by letting

$$
d^{*}(a, b)=\sum_{j=1}^{\infty} 2^{-j} D_{j}\left(a_{j}, b_{j}\right)
$$

for $a=\left(a_{1}, a_{2}, \ldots\right)$ and $b=\left(b_{1}, b_{2}, \ldots\right)$, then the inclusion map is an isometry of $(\bar{Q}, d)$ (and therefore also of $(X, d))$ into $\left.\left(Q, d^{*}\right)\right)$. Choose a point $q=\left(q_{1}, q_{2}, \ldots\right)$ in $Q$. We now define for each positive integer $i$ an isometry $H_{l}$ of $\left(Q_{i}, d_{l}\right)$ into $\left(Q, d^{*}\right)$ by letting

$$
H_{i}(x)=\left(p_{1 i}(x), \ldots, p_{l i}(x), q_{i+1}, q_{l+2}, \ldots\right)
$$

for every $x \in X_{i}$. We define $h_{i}$ as the restriction of $H_{l}$ on $X_{l}$ and we put $X_{i}^{*}=h_{i}\left(X_{i}\right)$. Note that for every $j \geq i$ there is a map $p_{i j}^{*}: X_{j}^{*} \rightarrow X_{i}^{*}$ given by

$$
\begin{aligned}
p_{l j}^{*}( & \left.\left(p_{1 j}(x), \ldots, p_{i j}(x), p_{l+1 j}(x), \ldots, p_{j j}(x), q_{J+1}, q_{J+2}, \ldots\right)\right) \\
& =\left(p_{1 i}\left(p_{l j}(x)\right), \ldots, p_{i i}\left(p_{l j}(x)\right), q_{i+1}, q_{l+2}, \ldots\right) \\
& =\left(p_{1 j}(x), \ldots, p_{i j}(x), q_{i+1}, q_{l+2}, \ldots\right)
\end{aligned}
$$

and that $d^{*}\left(p_{i j}^{*}\left(x_{j}^{*}\right), x_{j}^{*}\right)<2^{-i}$ for all $x_{j}^{*} \in X_{j}^{*}$. Also, observe that there is a map $p_{l}^{*}: X \rightarrow X_{i}^{*}$ defined by

$$
p_{i}^{*}\left(\left(x_{1}, x_{2}, x_{3}, \ldots\right)\right)=\left(x_{1}, x_{2}, \ldots, x_{i}, q_{i+1}, q_{i+2}, \ldots\right)
$$

and that

$$
d^{*}\left(p_{i}^{*}\left(x_{1}, x_{2}, \ldots\right),\left(x_{1}, x_{2}, \ldots\right)\right)<2^{-i}
$$

for all $\left(x_{1}, x_{2}, \ldots\right) \in X$.

In $\$ \S 4-6$ we shall always consider the spaces $X$ and $X_{i}$ with metrics $d$ and $d_{i}$, respectively. For example, in Definition (4.1) below, when we say that $p_{\imath j} \circ \psi$ is $\varepsilon$-close to $\varphi$ we mean that $d_{l}\left(p_{i j} \circ \psi, \varphi\right)<\varepsilon$.

4. Approximate absolute neighborhood retracts. Here we shall characterize surjective inverse $\mathrm{AANR}_{\mathrm{C}}$-sequences whose limits are $\mathrm{AANR}_{\mathrm{C}}$ 's and surjective inverse ANR-sequences whose limits are $\mathrm{AANR}_{\mathrm{N}}$ 's.

(4.1) Definition. An inverse sequence $\underline{X}=\left\{X_{i}, p_{\imath_{l}+1}\right\}$ is called (internally) $e$-movable provided for every $\varepsilon>0$ there is an index $i_{0}$ such that for every $i \geq i_{0}$, every $j \geq i$ and for every $\mathscr{P}_{\text {-map }} \varphi: K \rightarrow X_{l}$ there is a 
$\operatorname{map}\left(\psi: K \rightarrow X=\lim _{\leftarrow} \underline{X}\right) \psi: K \rightarrow X_{j}$ with $\left(p_{i} \circ \psi \varepsilon\right.$-close to $\left.\varphi\right) p_{i j} \circ \psi$ $\varepsilon$-close to $\varphi$.

(4.2) THEOREM. For a surjective inverse $A A N R_{\mathrm{C}}$-sequence $\underline{X}=$ $\left\{X_{i}, p_{i+1}\right\}$ the following are equivalent.

(i) $X=\lim \underline{X}$ is e-movable or, equivalently, an $A A N R_{\mathrm{C}}$.

(ii) $X$ is e-movable.

(iii) $\underline{X}$ is internally e-movable.

(iv) The sequence $\left\{X_{i}^{*}\right\}$ converges e-movably to $X$ [Č6].

(v) The sequence $\left\{X_{i}^{*}\right\}$ converges to $X$ in the metric of continuity $d_{c}^{*}$ induced on $2^{Q}$ by the metric $d^{*}$.

Proof. We shall prove (i) $\Rightarrow$ (ii), (ii) $\Rightarrow$ (iii), and (iii) $\Rightarrow$ (v). We already proved in [Č6] that (iv) and (v) are equivalent and that (iv) $\Rightarrow$ (i).

(i) $\Rightarrow$ (ii). Let an $\varepsilon>0$ be given. Since $X$ is an $e$-movable compactum in the Hilbert cube $Q$, by Proposition (4.2) in [ᄃ̌2], there is a neighborhood $V$ of $X$ in $Q$ such that for every neighborhood $W$ of $X$ in $Q$ and every $\mathscr{P}_{\text {-map }} \varphi: K \rightarrow V$ there is a map $\psi: K \rightarrow W$ which is $(\varepsilon / 3)$-close to $\varphi$. Select an index $i_{0}$ so that $X_{i}^{*} \subset V$ and so that $p_{i j}^{*}$ is an ( $\left.\varepsilon / 3\right)$-map and $p_{j}^{*}$ is an $(\varepsilon / 6)$-map for all $j \geq i \geq i_{0}$. Consider arbitrary indices $j \geq i \geq i_{0}$ and a $\rho_{\text {-map }} \varphi: K \rightarrow X_{i}$. Since $X_{j}$ is an $e$-movable compactum, there is a neighborhood $W$ of $X$ in $Q$ and an extension $\bar{p}_{j}^{*}: W \rightarrow X_{j}^{*}$ of $p_{j}^{*}$ such that

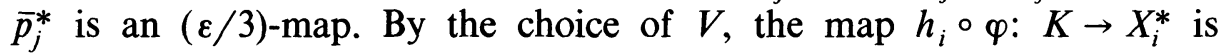
$(\varepsilon / 3)$-close to a map $\psi_{1}: K \rightarrow W$. But, then $\psi_{1}^{*}=\bar{p}_{j}^{*} \circ \psi_{1}$ is $(2 \varepsilon / 3)$-close to $h_{i} \circ \varphi$. Hence, $p_{i j}^{*} \circ \psi_{1}^{*}=p_{i j}^{*} \circ \bar{p}_{j}^{*} \circ \psi_{1}$ is $\varepsilon$-close to $h_{i} \circ \varphi$. It follows that $\varphi$ is $\varepsilon$-close to $p_{l j} \circ \psi$, where $\psi=h_{j}^{-1} \circ \bar{p}_{j}^{*} \circ \psi_{1}$, because $h_{i}$ is an isometry and the diagram

$$
\begin{array}{cccc}
X_{i}^{*} & \stackrel{p_{\imath \jmath}^{*}}{\leftarrow} & X_{j}^{*} \\
h_{i} \uparrow & & \uparrow h_{\jmath} \\
X_{i} & \stackrel{\leftarrow}{p_{\imath \jmath}} & X_{j}
\end{array}
$$

commutes.

(ii) $\Rightarrow$ (iii). It clearly suffices to prove that for every $\varepsilon>0$ there is an index $i_{0}$ such that for every $i \geq i_{0}$ there is an $\varepsilon$-map $f: X_{i}^{*} \rightarrow X$. For a given $\varepsilon>0$, pick an index $i_{0}$ so that for all $j \geq i \geq i_{0}$ there is a map $f_{i j}: X_{i} \rightarrow X_{j}$ with $p_{i j} \circ f_{i j}(\varepsilon / 2)$-close to $\mathrm{id}_{X_{i}}$. Let $i \geq i_{0}$ and select a sequence $i=i_{1}<$ $i_{2}<\cdots$ such that for every $j>0$ there is a map $f_{j}: X_{t_{j}} \rightarrow X_{i_{j+1}}$ with $p_{i, i_{j+1}} \circ f_{j}\left(\varepsilon / 2^{j}\right)$-close to id $X_{X_{i}}$ (with the distance measured with respect to 
the metric $d_{i_{j}}$ ). Then the map $f_{j}^{*}: X_{i_{j}}^{*} \rightarrow X_{i_{j+1}}^{*}$ defined by $f_{j}^{*}=h_{i_{j+1}} \circ f_{j} \circ h_{i_{j}}^{-1}$ is an $\left(\varepsilon / 2^{j}\right)$-map (measured in the metric $d^{*}$ ) for every $j>0$. Hence $f=\lim _{n \rightarrow \infty} f_{n}^{*} \circ f_{n-1}^{*} \circ \cdots \circ f_{1}^{*}$ is an $\varepsilon$-map of $X_{i}^{*}$ into $X$.

(iii) $\Rightarrow(v)$. By assumption, for every $\varepsilon>0$ there is an index $i_{0}$ such that for every $i \geq i_{0}$ there is a map $f_{i}: X_{i}^{*} \rightarrow X$ with $p_{i}^{*} \circ f_{i}(\varepsilon / 2)$-close to $\mathrm{id}_{X_{i}^{*}}$. But, if $i_{0}$ is so large that each $p_{i}^{*}$ is an ( $\left.\varepsilon / 2\right)$-map, $f_{i}$ will be an $\varepsilon$-map so that $d_{c}^{*}\left(X_{i}^{*}, X\right)<\varepsilon$ for all $i \geq i_{0}$.

(4.3) COROLLARY. If every bonding map $p_{i i+1}$ in an inverse $A A N R_{\mathrm{C}}$-sequence $\underline{X}=\left\{X_{i}, p_{i+1}\right\}$ is an approximately right invertible map [Ge1], $[\check{\mathbf{C}} \mathbf{1}]$, then $X=\lim \underline{X}$ is an e-movable compactum.

Proof. The assumption about bonding maps clearly implies that the inverse sequence $\underline{X}$ is $e$-movable so that we can apply (4.2)(ii) $\Rightarrow(\mathrm{i})$.

Since a compactum is an $A_{A N R}$ iff it is an $A_{A N R}$ and an FANR, [Bo], (1.2) and (4.2) imply the following.

(4.4) Corollary. Let $\underline{X}=\left\{X_{i}, p_{i+1}\right\}$ be a surjective ANR-sequence. The following are equivalent.

(i) $X=\lim \underline{X}$ is an $A A N R_{\mathrm{N}}$.

(ii) $\underline{X}$ is e-movable and strongly movable $[\mathbf{M}]$.

(iii) The sequence $X_{1}^{*}, X_{2}^{*}, \ldots$ converges both strongly movably regularly and e-movably to $X$.

5. Internally $e$-calm and e-calm compacta. In [Č1] the author defined internally $e$-calm and $e$-calm compacta in order to get a new characterization of ANR's analogous to the characterization of FANR's

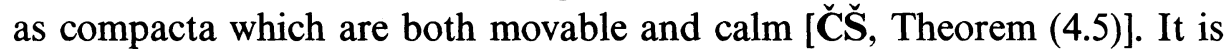
still unknown whether there exists an (internally) e-calm compactum which is not an ANR. This section shows how to recognize (internally) $e$-calm compacta as inverse limits of surjective inverse ANR-sequences.

(5.1) Definition. An inverse sequence $\underline{X}=\left\{X_{i}, p_{i+1}\right\}$ is internally $e$-calm if for every $\varepsilon>0$ there is an index $i$ and a $\delta>0$ such that for every index $j \geq i, p_{j} \circ \varphi$ and $p_{j} \circ \psi$ are $\varepsilon$-homotopic in $X_{j}$ whenever $\varphi$ and $\psi$ are $\delta$-close $\mathcal{P}$-maps into $X$.

(5.2) Definition. A sequence $\left\{A_{i}\right\}_{i=1}^{\infty}$ of compacta in a metric space $Y$ converges internally $e^{*}$-calmly to a compactum $A_{0}, A_{0} \subset Y$, provided in some, and hence in every, ANR $M$ which contains $Y$, for every $\varepsilon>0$ there 
is a $\delta>0$ such that for every neighborhood $W$ of $A_{0}$ in $M$ and every $\gamma>0$ there is an index $i_{0}$ so that $i \geq i_{0}$ implies $A_{i} \subset W$ and $\delta$-close $\mathscr{P}$-maps $\varphi, \psi$ : $K \rightarrow X$ are $\gamma$-close to maps $\varphi^{\prime}, \psi^{\prime}: K \rightarrow A_{i}$, respectively, which are $\varepsilon$-homotopic in $A_{i}$.

(5.3) THeOREM. For a surjective inverse ANR-sequence $\underline{X}=\left\{X_{i}, p_{i+1}\right\}$ the following are equivalent.

(i) $X=\lim \underline{X}$ is internally e-calm.

(ii) $X$ is internally e-calm.

(iii) The sequence $X_{1}^{*}, X_{2}^{*}, \ldots$ converges internally $e^{*}$-calmly to $X$.

Proof. (i) $\Rightarrow$ (ii). Let an $\varepsilon>0$ be given. Select an $i$ so big that $p_{j}^{*}$ is an ( $\varepsilon / 3$ )-map for all $j \geq i$ and a $\delta>0$ such that $\delta$-close $\mathcal{P}$-maps into $X$ are ( $\varepsilon / 3$ )-homotopic in every neighborhood of $X$ in $Q$. For $j \geq i$, choose a neighborhood $W_{j}$ of $X$ in $Q$ such that $p_{j}^{*}: X \rightarrow X_{j}^{*}$ extends to an ( $\left.\varepsilon / 3\right)$-map $\bar{p}_{j}^{*}: W_{j} \rightarrow X_{j}^{*}$. Now, $\delta$-close maps $\varphi, \psi: K \rightarrow X$ are $(\varepsilon / 3)$-homotopic in $W_{j}$. Hence, $\bar{p}_{j}^{*} \circ \varphi=p_{j}^{*} \circ \varphi$ and $\bar{p}_{j}^{*} \circ \psi=p_{j}^{*} \circ \psi$ are $\varepsilon$-homotopic in $X_{j}^{*}$ so that $p_{j} \circ \varphi=h_{j}^{-1} \circ p_{j}^{*} \circ \varphi$ is $\varepsilon$-homotopic to $p_{j} \circ \psi=h_{j}^{-1} \circ p_{j}^{*} \circ \psi$ (because $h$, is an isometry).

(ii) $\Rightarrow$ (iii). For a given $\varepsilon>0$, pick a $\delta>0$ and an index $i_{1}$ such that $p_{i} \circ \varphi$ and $p_{i} \circ \psi$ are $\varepsilon$-homotopic in $X_{i}\left(i \geq i_{1}\right)$ whenever $\varphi$ and $\psi$ are

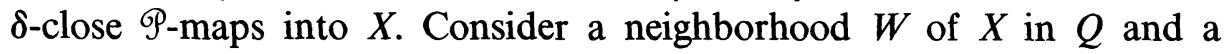
$\gamma>0$. Choose an index $i_{0} \geq i_{1}$ so that $p_{i}^{*}$ is a $\gamma$-map and $X_{i}^{*} \subset W$ for all $i \geq i_{0}$.

(iii) $\Rightarrow$ (i). Let an $\varepsilon>0$ be given. Select a $\delta>0$ with respect to $\varepsilon / 3$ using the assumption. Let $W$ be a compact ANR neighborhood of $X$ in $Q$ and let $\gamma \in \Gamma(W, \varepsilon / 3)$. Choose an index $i_{0}$ so that $X_{i_{0}}^{*} \subset W$ and so that $\delta$-close $\mathcal{P}$-maps $\varphi, \psi: K \rightarrow X$ are $\gamma$-close to maps $\varphi^{\prime}, \psi^{\prime}: K \rightarrow X_{i_{0}}^{*}$, respectively, with $\varphi^{\prime}$ and $\psi^{\prime}(\varepsilon / 3)$-homotopic in $W$. Since $\varphi$ and $\varphi^{\prime}$ are $(\varepsilon / 3)$-homotopic in $W$ and $\psi$ and $\psi^{\prime}$ are $(\varepsilon / 3)$-homotopic in $W$, it follows that $\varphi$ and $\psi$ are $\varepsilon$-homotopic in $W$ and therefore that $X$ is internally $e$-calm.

(5.4) Definition. An inverse sequence $\underline{X}=\left\{X_{i}, p_{i+1}\right\}$ is e-calm if for every $\varepsilon>0$ there is an index $i$ and a $\delta>0$ such that for every $j \geq i$ there is a $k \geq j$ with $p_{j k} \circ \varphi \varepsilon$-homotopic to $p_{j k} \circ \psi$ in $X_{j}$ whenever $\varphi$ and $\psi$ are $\delta$-close $\mathscr{P}$-maps into $X_{k}$.

(5.5) Definition. A sequence $\left\{A_{i}\right\}_{i=1}^{\infty}$ of compacta in a metric space $Y$ converges $e^{*}$-calmly to a compactum $A_{0}, A_{0} \subset Y$, provided in some, and hence in every, ANR $M$ which contains $Y$, for every $\varepsilon>0$ there is a 
$\delta>0$ such that for every neighborhood $W$ of $A_{0}$ in $M$ there is an index $i_{W}$ with the property that for every $i \geq i_{W}$ there is a neighborhood $W_{0}^{i}$ of $A_{i}$ in

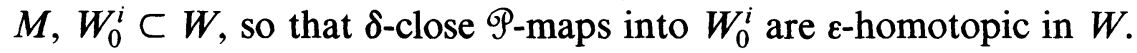

(5.6) ThEOREM. Let $\underline{X}=\left\{X_{i}, p_{i+1}\right\}$ be a surjective inverse ANR-sequence. The following are equivalent.

(i) $X=\lim \underline{X}$ is e-calm.

(ii) $\underline{X}$ is e-calm.

(iii) The sequence $X_{1}^{*}, X_{2}^{*}, \ldots$ converges $e^{*}$-calmly to $X$.

Proof. (i) $\Rightarrow$ (ii). For a given $\varepsilon>0$, select a neighborhood $V$ of $X$ in $Q$ and a $\delta>0$ such that $\mathscr{P}_{h}^{\varepsilon / 9}(V, \delta ; X)$ holds. Then choose an index $i$ so that $X_{j}^{*} \subset V$ and $p_{j}^{*}$ is an ( $\left.\varepsilon / 9\right)$-map of $X$ onto $X_{j}^{*}$ for all $j \geq i$. Let $j \geq i$. Extend $p_{j}^{*}$ to an ( $\left.\varepsilon / 9\right)$-map $\bar{p}_{j}^{*}: W \rightarrow X_{j}^{*}$ of a closed neighborhood $W$ of $X$ in $Q$ and let $\eta \in \Lambda\left(\bar{p}_{j}^{*}, \gamma\right)$, where $\gamma \in \Gamma\left(X_{j}^{*}, \varepsilon / 3\right)$. Inside $W \cap V$ pick a neighborhood $W_{0}$ of $X$ in $Q$ using $\mathscr{P}_{h}^{\varepsilon / 9}(V, \delta ; X)$ and take a $k \geq j$ so that $X_{k}^{*} \subset W_{0}$ and so that $p_{k}^{*}$ is an $\eta$-map.

Consider $\delta$-close $\mathscr{P}^{\mathcal{P}}$-maps $\varphi, \psi: K \rightarrow X_{k}$ into $X_{k}$. The compositions $h_{k} \circ \varphi$ and $h_{k} \circ \psi$ are $\delta$-close maps of $K$ into $W_{0}$. Hence, they are $(\varepsilon / 9)$-homotopic in $W$. Since $\bar{p}_{j}^{*}$ is an ( $\left.\varepsilon / 9\right)$-map, $\bar{p}_{j}^{*} \circ h_{k} \circ \varphi$ and $\bar{p}_{j}^{*} \circ h_{k} \circ \psi$ are $(\varepsilon / 3)$-homotopic in $X_{j}^{*}$. But, for every point $y \in X_{k}^{*}$, there is $x \in X$ such that $p_{k}^{*}(x)=y$ so that $\bar{p}_{j}^{*}(x)=p_{j}^{*}(x)=p_{j k}^{*} \circ p_{k}^{*}(x)=$ $p_{j k}^{*}(y)$ and $\bar{p}_{j}^{*}(y)$ are $\gamma$-close. It follows that $p_{j k}^{*} \circ h_{k} \circ \varphi$ is $(\varepsilon / 3)$-homotopic to $\bar{p}_{j}^{*} \circ h_{k} \circ \varphi$ in $X_{j}^{*}$. Thus, $p_{j k}^{*} \circ h_{k} \circ \varphi$ and $p_{j k}^{*} \circ h_{k} \circ \psi$ are $\varepsilon$-homotopic in $X_{j}^{*}$. This implies that $p_{j k} \circ \varphi$ and $p_{j k} \circ \psi$ are $\varepsilon$-homotopic in $X_{j}$.

(ii) $\Rightarrow$ (iii). Let $\varepsilon>0$. Choose an $i$ and a $\delta>0$ such that $i$ and $3 \delta$ satisfy (5.4) with respect to $\varepsilon / 3$. Consider a compact ANR neighborhood $W$ of $X$ in $Q$ and let $\eta \in \Gamma(W, \varepsilon / 9)$. Pick an index $j=i_{W} \geq i$ such that $X_{k}^{*} \subset W$ and $p_{j k}^{*}$ is an $\eta$-map for all $k \geq j$. Finally, for every $k \geq j$, let $W_{0}^{k}$ denote a neighborhood of $X_{k}^{*}$ in $W$ such that there is a $\min \{\delta, \eta\}$-map $r_{k}$ : $W_{0}^{k} \rightarrow X_{k}^{*}$.

(iii) $\Rightarrow$ (i). For a given $\varepsilon>0$, select a $\delta>0$ with respect to $\varepsilon / 3$ using (iii). Let $W$ be a compact ANR neighborhood of $X$ in $Q$ and let $\eta \in \Gamma(W, \varepsilon / 3)$. Then choose an $i \geq i_{W}$ and a neighborhood $W_{0}$ of $X$ in $Q$, $W_{0} \subset W$, such that $p_{i}^{*}$ extends to a $\min \{\delta / 3, \eta\}$-map $\bar{p}_{i}^{*}$ of $W_{0}$ into $W_{0}^{i}$. It can be easily checked that $(\delta / 3)$-close $\mathscr{P}$-maps into $W_{0}$ are $\varepsilon$-homotopic in

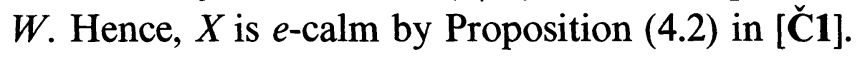


6. ANR's and $L C^{n}$ compacta. In this section we shall put together results from $\S \S 4$ and 5 and get conditions which characterize surjective inverse ANR-sequences whose inverse limits are ANR's. Then we shall indicate changes that one must make in our theorem to obtain analogous results for surjective inverse $L C^{n}$-sequences whose inverse limits are $L C^{n}$-compacta. In particular, the Fort-Segal characterization mentioned in the introduction appears as the 0 -dimensional case of ours. Some applications of our method are also presented.

(6.1) Definition. An inverse sequence $\underline{X}=\left\{X_{i}, p_{i+1}\right\}$ is strongly $e$-movable provided for every $\varepsilon>0$ there is an index $i_{0}$ such that for every $i \geq i_{0}$, for every $j \geq i$, and every $\delta>0$ there is a $k \geq j$ with the property that for every $\mathscr{P}_{p}$-pair $\left(K, K_{0}\right)$ and maps $\varphi: K \rightarrow X_{i}$ and $\psi_{0}: K_{0} \rightarrow X_{k}$ with $\varphi \mid K_{0}=p_{i k} \circ \psi_{0}$, there is a map $\psi: K \rightarrow X_{j}$ such that $p_{i j} \circ \psi$ is $\varepsilon$-close to $\varphi$ and $\psi \mid K_{0}$ is $\delta$-close to $p_{j k} \circ \psi_{0}$.

(6.2) Definition. A sequence $\left\{A_{i}\right\}_{i=1}^{\infty}$ of compacta in a metric space $Y$ converges strongly $e^{*}$-movably to a compactum $A_{0}, A_{0} \subset Y$, provided in some, and hence in every, ANR $M$ which contains $Y$, for every neighborhood $U$ of $A_{0}$ in $M$ and every $\varepsilon>0$ there is a neighborhood $V$ of $A_{0}$ in $M$, $V \subset U$, such that for every neighborhood $W$ of $A_{0}$ in $M$ there is an index $i_{W}$ with the property that for every $i \geq i_{W}$ there is a neighborhood $W_{0}^{i}$ of $A_{i}$ in $M, W_{0}^{i} \subset V \cap W$, so that for every $\mathscr{P}_{p}$-map $f:\left(K, K_{0}\right) \rightarrow\left(V, W_{0}^{i}\right)$ there is an $\varepsilon$-homotopy $f_{t}: K \rightarrow U, 0 \leq t \leq 1$, with $f_{0}=f, f_{1}(K) \subset W$, and $f_{1}\left|K_{0}=f\right| K_{0}$.

(6.3) ThEOREM. For a surjective inverse ANR-sequence $\underline{X}=\left\{X_{i}, p_{i i+1}\right\}$ the following are equivalent.

(i) $X=\lim \underline{X}$ is an $A N R$.

(ii) $X$ is strongly e-movable.

(iii) $\underline{X}$ is both e-movable and internally e-calm.

(iv) $\underline{X}$ is both e-movable and e-calm.

(v) The sequence $X_{1}^{*}, X_{2}^{*}, \ldots$ converges strongly $e^{*}$-movably to $X$.

(vi) The sequence $X_{1}^{*}, X_{2}^{*}, \ldots$ converges both e-movably and internally $e^{*}$-calmly to $X$.

(vii) The sequence $X_{1}^{*}, X_{2}^{*}, \ldots$ converges both e-movably and $e^{*}$-calmly to $X$.

Proof. We shall prove that (i) $\Rightarrow$ (ii) and (ii) $\Rightarrow$ (iv). The other implications are consequences of results in $\$ \S 4$ and 5 and Theorem (4.9)(a) and Lemma (4.10) in [Č1]. 
(i) $\Rightarrow$ (ii). For a given $\varepsilon>0$, select a compact ANR neighborhood $V$ of $X$ in $Q$ such that for every neighborhood $W$ of $X$ in $Q$ there is a neighborhood $W_{0}$ of $X$ in $Q, W_{0} \subset V \cap W$, with the property that every $\mathscr{\rho}_{p}$-map $\varphi_{1}^{*}:\left(K, K_{0}\right) \rightarrow\left(V, W_{0}\right)$ is $(\varepsilon / 4)$-close to a map $\varphi_{2}^{*}: K \rightarrow W$ which

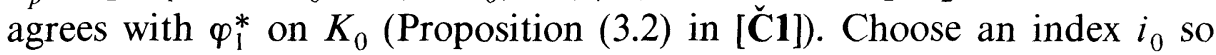
that $X_{i}^{*} \subset V$ and $p_{l j}^{*}$ and $p_{j}^{*}$ are $(\eta / 2)$-maps for all $i$ and $j, j \geq i \geq i_{0}$, where $\eta \in \Gamma(V, \varepsilon / 4), 0<\eta<\varepsilon / 4$.

Consider indices $j$ and $i, j \geq i \geq i_{0}$, and a $\delta>0$. Choose an ( $\left.\eta / 2\right)$-map $r: W \rightarrow X$ which retracts a neighborhood $W$ of $X$ in $Q$ onto $X$. Inside the intersection $V \cap W$ pick a neighborhood $W_{0}$ of $X$ in $Q$ as above and take an index $k \geq j$ such that $X_{k}^{*} \subset W_{0}$ and such that $r \mid X_{k}^{*}$ and $p_{k}^{*}$ are $(\gamma / 2)$-maps where $2 \gamma \in \Lambda\left(p_{J}^{*}, \delta\right)$ and $0<\gamma<\varepsilon / 4$. Observe that $\gamma \in$ $\Lambda\left(p_{\jmath k}^{*}, \delta\right)$ because $p_{k}^{*}$ is onto and $p_{\jmath}^{*}=p_{\jmath k}^{*} \circ p_{k}^{*}$.

Let $\left(K, K_{0}\right)$ be a pair in $\mathscr{P}_{p}$ and let $\varphi: K \rightarrow X_{l}$ and $\psi_{0}: K_{0} \rightarrow X_{k}$ be maps with $\varphi \mid K_{0}=p_{\imath k} \circ \psi_{0}$. The compositions $\varphi^{*}=h_{l} \circ \varphi$ and $\psi_{0}^{*}=$ $h_{k} \circ \psi_{0}$ satisfy $\varphi^{*} \mid K_{0}=p_{i k}^{*} \circ \psi_{0}^{*}$. Since $p_{l k}^{*}$ is an $(\eta / 2)$-map, there is an $(\varepsilon / 4)$-homotopy $g_{t}: K_{0} \rightarrow V, 0 \leq t \leq 1$, such that $g_{0}=\varphi^{*} \mid K_{0}=p_{\imath k}^{*} \circ \psi_{0}^{*}$ and $g_{1}=\psi_{0}^{*}$. By the homotopy extension theorem, $g_{1}$ can be extended to a map $\varphi_{1}^{*}: K \rightarrow V$ which is $(\varepsilon / 4)$-close to $\varphi^{*}$. The choice of $V$ and $W_{0}$ implies that $(\varepsilon / 4)$-close to $\varphi_{1}^{*}$ there is a map $\varphi_{2}^{*}: K \rightarrow W$ which agrees with $\varphi_{1}^{*}$ on $K_{0}$. Let $\psi^{*}=p_{J}^{*} \circ r \circ \varphi_{2}^{*}$ and let $\psi=h_{J}^{-1} \circ p_{J}^{*} \circ r \circ \varphi_{2}^{*}$. Since $r$ and $p_{J}^{*}$ are $(\varepsilon / 8)$-maps and $p_{l j}^{*}$ is an $(\varepsilon / 4)$-map, $\psi^{*}$ is $(\varepsilon / 4)$-close to $\varphi_{2}^{*}$ and $p_{\imath j}^{*} \circ \psi^{*}$ is $\varepsilon$-close to $\varphi^{*}$ and hence $p_{l j} \circ \psi$ is $\varepsilon$-close to $\varphi$. On the other hand, $p_{k}^{*} \circ r \circ \psi_{0}^{*}$ is $\gamma$-close to $\psi_{0}^{*}$ so that $p_{J k}^{*} \circ p_{k}^{*} \circ r \circ \psi_{0}^{*}=p_{j}^{*} \circ r \circ \psi_{0}^{*}=$ $\psi^{*} \mid K_{0}$ is $\delta$-close to $p_{j k}^{*} \circ \psi_{0}^{*}$ and thus $p_{J k} \circ \psi_{0}$ is $\delta$-close to $\psi \mid K_{0}$.

(ii) $\Rightarrow$ (iv). Since every strongly $e$-movable ANR-sequence $\underline{X}$ is clearly $e$-movable, we must show that $\underline{X}$ is $e$-calm. For an $\varepsilon>0$, pick an index $i_{0}$ as in Definition (6.1) but with respect to $\varepsilon / 9$. Then choose an $i \geq i_{0}$ so that $p_{I j}^{*}$ is an ( $\left.\varepsilon / 9\right)$-map for every $j \geq i$. Let a $\delta>0$ have the property that $3 \delta \in \Lambda\left(p_{i}, \eta\right)$ where $\eta \in \Gamma\left(X_{i}, \varepsilon / 9\right)$.

Consider an index $j \geq i$ and select a $k \geq j$ as in Definition (6.1) with respect to $j$ and a $\gamma \in \Gamma\left(X_{\jmath}, \varepsilon / 3\right)$ and so that $p_{k}^{*}$ is a $\delta$-map. Let $\varphi, \psi$ : $K \rightarrow X_{k}$ be $\delta$-close maps of a compactum $K$ into $X_{k}$. Then $\varphi^{*}=h_{k} \circ \varphi$ : $K \rightarrow X_{k}^{*}$ and $\psi^{*}=h_{k} \circ \psi: K \rightarrow X_{k}$ are $\delta$-close and for every $x \in K$ there are $y, z \in X$ such that $\varphi^{*}(x)=p_{k}^{*}(y)$ and $\psi^{*}(x)=p_{k}^{*}(z)$. Since $y$ and $z$ are $3 \delta$-close, $p_{i}^{*}(y)$ and $p_{i}^{*}(z)$ are $\eta$-close. But $p_{l}^{*}(y)=p_{l k}^{*} \circ p_{k}^{*}(y)=$ $p_{i k}^{*}\left(\varphi^{*}(x)\right)$ and $p_{i}^{*}(z)=p_{i k}^{*} \circ p_{k}^{*}(z)=p_{i k}^{*}\left(\psi^{*}(x)\right)$ so that $p_{l k}^{*} \circ \varphi^{*}$ and $p_{i k}^{*} \circ \psi^{*}$ are $\eta$-close maps into $X_{l}^{*}$. Hence, there is an ( $\left.\varepsilon / 9\right)$-homotopy $H$ : $K \times I \rightarrow X_{i}^{*}$ with $H_{0}=p_{\imath k}^{*} \circ \varphi^{*}$ and $H_{1}=p_{\imath k}^{*} \circ \psi^{*}$. The choice of $k$ 
implies that there is a map $G: K \times I \rightarrow X_{j}^{*}$ such that $p_{i j}^{*} \circ G$ is $(\varepsilon / 9)$-close to $H$ and $p_{j k}^{*} \circ \varphi^{*}$ is $\gamma$-close to $G_{0}$ and $p_{j k}^{*} \circ \psi^{*}$ is $\gamma$-close to $G_{1}$. Since $H$ is an ( $\varepsilon / 9)$-homotopy and $p_{i j}^{*}$ is an ( $\left.\varepsilon / 9\right)$-map, $G$ is an ( $\left.\varepsilon / 3\right)$-homotopy. On the other hand, the selection of $\gamma$ insures that $p_{j k}^{*} \circ \varphi^{*}$ is $(\varepsilon / 3)$-homotopic to $G_{0}$ and that $p_{j k}^{*} \circ \psi^{*}$ is $(\varepsilon / 3)$-homotopic to $G_{1}$. Hence, $p_{j k}^{*} \circ \varphi^{*}$ and $p_{j k}^{*} \circ \psi^{*}$ are $\varepsilon$-homotopic (in $X_{j}^{*}$ ) so that $p_{j k} \circ \varphi$ and $p_{j k} \circ \psi$ are $\varepsilon$-homotopic (in $X_{j}$ ).

(6.4) REMARK. In condition (v) in the above theorem the strongly $e^{*}$-movable convergence cannot be replaced by the more restrictive strongly $e$-movable convergence [Č7]. Indeed, by [MS1, Theorem 1 and Example 9], the simple closed curve $X$ can be represented as an inverse limit of a surjective ANR-sequence $\underline{X}=\left\{X_{i}, p_{i+1}\right\}$ where each $X_{i}$ is the 2-dimensional torus. If the sequence $\left\{X_{i}^{*}\right\}$ were to converge strongly

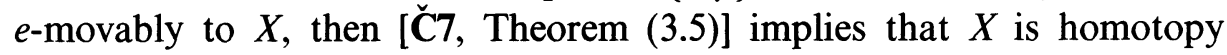
equivalent to almost all $X_{i}$, an obvious contradiction.

However, with an additional assumption that every bonding map $p_{i+1}$ is ARI, the characterization involving strongly $e$-movable convergence holds.

(6.5) THEOREM. Let $\underline{X}=\left\{X_{i}, p_{i+1}\right\}$ be a surjective inverse ANR-sequence and assume that each map $p_{i i+1}$ is $A R I$. Then $X=\lim \underline{X}$ is an $A N R$ iff the sequence $\left\{X_{i}^{*}\right\}$ converges strongly e-movably to $X$.

Proof. Suppose that $X$ is an ANR. By (6.3) and (4.2), $\underline{X}$ is strongly

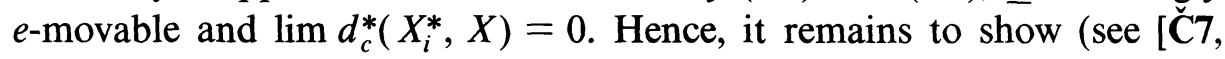
Theorem (3.10)]) that for every $\varepsilon>0$ there is an index $i$ and a $\delta>0$ such

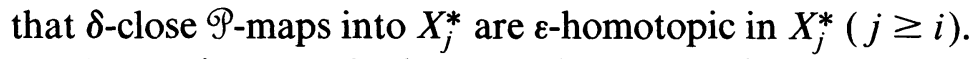

For a given $\varepsilon>0$, choose an index $i_{0}$ so large that $p_{i j}^{*}$ is an ( $\left.\varepsilon / 9\right)$-map for all $i$ and $j, j \geq i \geq i_{0}$, and that (6.1) holds with respect to $\varepsilon / 9$ and $i_{0}$. Let $\delta>0$ satisfy $3 \delta \in \Lambda\left(p_{i_{0}}^{*}, \eta\right)$, where $\eta \in \Gamma\left(X_{i_{0}}^{*}, \varepsilon / 27\right)$, and let $i \geq i_{0}$ be such that $p_{j}^{*}$ is a $\delta$-map for all $j \geq i$. Observe that $\delta \in \Lambda\left(p_{i_{0} j}^{*}, \eta\right)$ for all $j \geq i$.

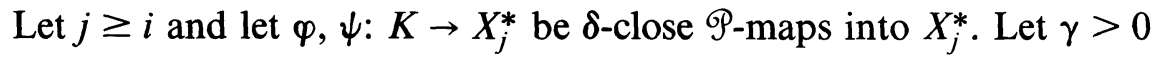
be such that $\gamma \in \Lambda\left(p_{i_{0}}^{*}, \eta\right)$ and $\gamma \in \Gamma\left(X_{j}^{*}, \varepsilon / 6\right)$. Pick $k \geq j$ with respect to $\gamma$ and $j$ using the way in which $i_{0}$ was chosen. Since $p_{j k}^{*}$ is an ARI map, there are maps $\varphi^{\prime}, \psi^{\prime}: K \rightarrow X_{k}^{*}$ with $p_{j k}^{*} \circ \varphi^{\prime} \gamma$-close to $\varphi$ and $p_{j k}^{*} \circ \psi^{\prime}$ $\gamma$-close to $\psi$. Hence, there is an ( $\varepsilon / 9)$-homotopy $H: K \times I \rightarrow X_{i_{0}}^{*}$ joining $p_{i_{0} k}^{*} \circ \varphi^{\prime}$ and $p_{i_{0} k}^{*} \circ \psi^{\prime}$. On the other hand, $p_{j k}^{*} \circ \varphi^{\prime}$ and $\varphi$ are $(\varepsilon / 6)$-homotopic in $X_{j}^{*}$ and $p_{j k}^{*} \circ \psi^{\prime}$ and $\psi$ are $(\varepsilon / 6)$-homotopic in $X_{j}^{*}$. Choose $G$ : $K \times I \rightarrow X_{j}^{*}$ such that $p_{i_{0} j}^{*} \circ G$ is $(\varepsilon / 9)$-close to $H$ and $G_{0}$ is $\gamma$-close to 
$p_{j k}^{*} \circ \varphi^{\prime}$ and $G_{1}$ is $\gamma$-close to $p_{j k}^{*} \circ \psi^{\prime}$. Clearly, $G$ is an $(\varepsilon / 3)$-homotopy so that $\varphi$ and $\psi$ are $\varepsilon$-homotopic in $X_{j}^{*}$.

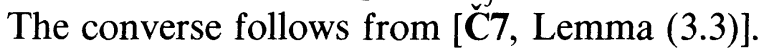

(6.6) Corollary. If the inverse limit $X$ of an inverse ANR-sequence $\underline{X}=\left\{X_{l}, p_{t i+1}\right\}$ with $A R I$ bonding maps is an ANR, then $X$ is (simple) homotopy equivalent to almost all bonding spaces $X_{l}$.

Proof. Combine (6.5) and [Č 7, Theorem (6.1)].

In order to handle $L C^{n}$ compacta, we define notions of $e$ - $n$-movability and (internal) $e$ - $n$-calmness for an inverse sequence and notions of $e-n$ movable and (internally) $e^{*}$ - $n$-calm convergence for sequences of compacta in a metric space simply by restricting $K$ in Definitions (4.1), (5.1), (5.4), (5.2), and (5.5), respectively, to compact ANR's of dimension $\leq n$. Similarly, if we require in Definitions (6.1) and (6.2) that $\left(K, K_{0}\right)$ is a pair of at most $n$-dimensional ANR's, we get notions of strong $e$ - $n$-movability (for inverse sequences) and strong $e^{*}-n$-movable convergence.

Consistent changes from arbitrary ANR's to ANR's of dimension $\leq n$ and from ANR's to $L C^{n}$ compacta in our proofs provide the proof of the following.

(6.7) THEOREM. For a surjective inverse $L C^{n}$-sequence $\underline{X}=\left\{X_{i}, p_{i_{1}+1}\right\}$ the following are equivalent.

(i) $X=\lim \underline{X}$ is an LC $C^{n}$ compactum.

(ii) $X$ is strongly e- $(n+1)$-movable.

(iii) $\underline{X}$ is both $e-(n+1)$-movable and (internally) $e-(n+1)$-calm.

(iv) The sequence $X_{1}^{*}, X_{2}^{*}, \ldots$ converges strongly $e^{*}-(n+1)$-movably to $X$.

(v) The sequence $X_{1}^{*}, X_{2}^{*}, \ldots$ converges both $e-(n+1)$-movably and (internally) $e^{*}-(n+1)$-calmly to $X$.

There are also versions of (6.4), (6.5), and (6.6) for $L C^{n}$ compacta. However, for $n=0$ the assumptions in (6.5) is not necessary (we can always get maps $\varphi^{\prime}$ and $\psi^{\prime}$ because without loss of generality $K$ can be chosen a single point and $p_{\imath j}$ 's are onto). Since strongly $e$-1-movable convergence is clearly equivalent to 0 -regular convergence [Wh] for locally connected compacta (see Theorem (3.10) in [Č 7]), it follows that (6.7) includes Theorem 3 in [FS] as a special case.

Another interesting consequence of the method of proof of Theorem (6.3) is the following improvement of Geoghegan's Theorem (1.3) in [Ge2]. 
(6.8) THEOREM ([Ge2]). Let $\underline{X}=\left\{X_{i}, p_{i+1}\right\}$ be an inverse ANR-sequence with each bonding map $p_{i+1}$ an approximate fibration [CD]. Then $X=\lim \underline{X}$ is an ANR iff $X$ is an FANR.

Proof. Since every ANR is an FANR, it remains to prove that if $X$ is an FANR (which is equivalent to Geoghegan's condition that $X$ is shape equivalent to a $\mathrm{CW}$-complex) then it must be an ANR. The approximate homotopy lifting property of approximate fibrations [CD] and the proof

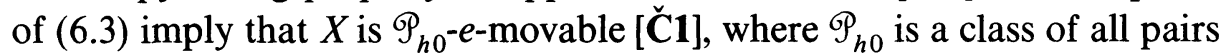
( $K \times[0,1], K \times\{0\})$ with $K \in \mathscr{P}$. In other words, for every neighborhood $U$ of $X$ in $Q$ and every $\varepsilon>0$ there is a neighborhood $V$ of $X$ in $Q$, $V \subset U$, such that for every neighborhood $W$ of $X$ in $Q$ there is a neighborhood $W_{0}$ of $X$ in $Q, W_{0} \subset W \cap V$, with the property that for every pair $\left(K, K_{0}\right) \in \mathscr{P}_{h 0}$ and a map $f:\left(K, K_{0}\right) \rightarrow\left(V, W_{0}\right)$ there is an $\varepsilon$-homotopy $f_{t}: K \rightarrow U, 0 \leq t \leq 1$, with $f_{0}=f, f_{1}(K) \subset W$, and $f_{1} \mid K_{0}=$ $f \mid K_{0}$. Hence, the theorem follows from the following theorem which gives a new characterization of compact ANR's.

(6.9) TheOrem. A compactum $X$ is an ANR iff it is both an FANR and $\mathscr{P}_{h 0}$-e-movable.

Proof. Every ANR is clearly an FANR and $\mathscr{P}_{h 0^{-}}$-e-movable. Conversely, suppose $X$ is a $\mathscr{P}_{h 0^{-}} e$-movable FANR. We shall prove that $X$ is strongly $e$-movable. Consider $X$ as a subset of the Hilbert cube $Q$ and let a compact ANR neighborhood $U$ of $X$ in $Q$ and an $\varepsilon>0$ be given. Let $\eta \in \Gamma^{*}(U, \varepsilon / 2), 0<\eta<\varepsilon / 2$. Choose a neighborhood $V_{1}$ of $X$ in $Q$, $V_{1} \subset U$, with respect to $U$ and $\eta$ using the fact that $X$ is $\mathscr{P}_{h 0^{-e}}$-movable. The pick a neighborhood $V$ of $X$ in $Q, V \subset V_{1}$, such that for every neighborhood $W$ of $X$ in $Q$, there is a neighborhood $W_{0}$ of $X$ in $Q$, $W_{0} \subset V \cap W$, with the property that for every $\mathscr{P}_{p}$-map $f:\left(K, K_{0}\right) \rightarrow$ $\left(V, W_{0}\right)$ there is a homotopy $f_{t}: K \rightarrow V_{1}, 0 \leq t \leq 1$, with $f_{0}=f, f_{1}(K) \subset W$, and $f_{t}\left|K_{0}=f\right| K_{0}$ for all $t \in[0,1]$ (this requires $X$ to be a pointed FANR which follows either directly from Hastings-Heller's theorem [HH] or one can easily verify that $X$ is arcwise connected and thus pointed 1-movable [KM]). We claim that $\mathscr{P}_{p}^{\varepsilon}(U, V ; X)$ holds.

Indeed, let $W$ be an arbitrary neighborhood of $X$ in $Q$. Inside $V \cap W$ pick a neighborhood $\bar{W}_{0}$ of $X$ in $Q$ using the choice of $V_{1}$ and then a smaller neighborhood $W_{0}$ of $X$ in $Q$ with respect to $\bar{W}_{0}$ using the way in which $V$ was chosen. If $f:\left(K, K_{0}\right) \rightarrow\left(V, W_{0}\right)$ is a $\mathscr{P}_{p}$-map, then there is a homotopy $F: K \times[0,1] \rightarrow V_{1}$ with $F_{0}=f, F_{1}(K) \subset \bar{W}_{0}$ and $F_{t}\left|K_{0}=f\right| K_{0}$ 
for all $t \in[0,1]$. But, we know that $\eta$-close to $F$ there is a map $G$ : $K \times[0,1] \rightarrow W$ with $G_{1}=F_{1}$. Clearly, $G \mid K_{0} \times[0,1]$ is an $\eta$-homotopy between $G_{0} \mid K_{0}$ and $G_{1}\left|K_{0}=f\right| K_{0}$ while $G_{0}$ and $F_{0}$ are $(\varepsilon / 2)$-homotopic in $U$ via a homotopy which agrees with $G_{1-t}$ on $K_{0}$. Hence, by the homotopy extension theorem, $F_{0}$ is $\varepsilon$-homotopic in $U$ to a map of $K$ into $W$ that equals $f \mid K_{0}$ on $K_{0}$.

Theorem (6.8) has an amusing corollary which to the best of my knowledge has not appeared in the literature.

(6.10) COROllaRY. A compact metrizable topological group $G$ is a Lie group iff $G$ is an FANR.

Proof. By results in [Sz], a compact metrizable topological group $G$ can be represented as the inverse limit of an inverse sequence $\underline{X}=$ $\left\{X_{l}, p_{t \imath+1}\right\}$ where each bonding space $X_{i}$ is a manifold and each bonding map $p_{t i+1}$ is a locally trivial fibre map. Theorem (6.8) implies that $G$ will be an ANR iff $G$ is an FANR. Hence, the theorem follows from the corollary to Theorem 4 in [Sz].

The next application of our methods was also observed by McAuley and Robinson [McR].

(6.11) Corollary. Let $\underline{X}=\left\{X_{l}, p_{l_{l}+1}\right\}$ be an inverse $L C^{n}$-sequence with each bonding map $p_{t_{l+1}}$ an $U V^{n}$-map (or, equivalently, a $\Sigma^{n}$-trivial map [Č́1]). Then $X=\lim \underline{X}$ is an LC $C^{n}$ compactum.

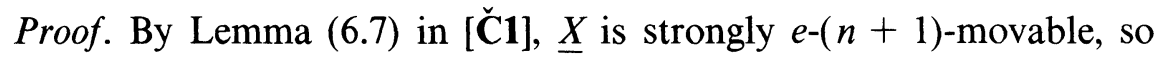
that $X$ is an $L C^{n}$ compactum by Theorem (6.7).

7. Dimension. In this final section we shall give a new characterization of inverse $\mathrm{AANR}_{\mathrm{C}}$-sequences whose inverse limits have dimension $\leq n$. As in the previous sections, the idea is the same, namely, to "rigidify" the corresponding result (1.4) in shape theory. However, the technique of proof differs from the one used in $\$ \S 4-6$ (that relied heavily on Fort-Segal embeddings and remetrizations of bonding spaces and the inverse limit) and utilize the following improvement of Lemma 1 in [MR]. In view of Theorem 8 in [M2], this is a special case of Proposition 1 in [M2].

(7.1) LEMMA. Let $\underline{X}=\left\{X_{i}, p_{i+1}\right\}$ be an inverse sequence of compacta and let $Y$ be an $A A N R_{\mathrm{C}}$. Then the following assertions hold:

(i) For every $\varepsilon>0$ and for every map $f: X \rightarrow Y$ there is an $i^{*}$ such that for each $i \geq i^{*}$ there is a map $f_{i}: X_{i} \rightarrow Y$ with $d\left(f_{i}^{\circ} p_{i}, f\right)<\varepsilon$. 
(ii) If $\varepsilon>0$ and $f_{t}, g_{t}: X_{t} \rightarrow Y$ are maps such that $d\left(f_{l}^{\circ} \circ p_{t}, g_{i} \circ p_{t}\right)<\varepsilon$, then there is an $i^{*} \geq i$ such that $d\left(f_{i} \circ p_{i j}, g_{i} \circ p_{i j}\right)<\varepsilon$ for every $j \geq i^{*}$.

Proof. Since the proof of Lemma 1(ii) in [MR] does not use any assumptions on $Y$, it remains only to prove (i).

Let $X^{*}$ denote a compactum described in the proof of Lemma 1 in [MR]. Recall that as a set $X^{*}$ in the disjoint union $X \cup\left(\cup_{l}>0 X_{l}\right)$ and that the basis for the topology of $X^{*}$ is given by open subsets $U_{t} \subseteq X_{t}$ and by the sets $U_{l}^{*}=p_{i}^{-1}\left(U_{l}\right) \cup \cup_{j \geq l} p_{i j}^{-1}\left(U_{l}\right)$. In this topology on $X^{*}$ both $X_{i}$ and $X$ inherit their original topologies, every neighborhood of $X$ in $X^{*}$ contains almost all $X_{i}$ 's, and for every $\varepsilon>0$ there is an index $i$ such that $d\left(p_{j}(x), x\right)<\varepsilon$ for all $j \geq i$ and $x \in X$.

Consider $Y$ as a subset of $Q$ and pick a compact ANR neighborhood $V$ of $Y$ in $Q$ for which there is an ( $\varepsilon / 2)$-map $r: V \rightarrow Y$ [C1]. Extend $f$ : $X \rightarrow Y$ to a map $f^{*}: U \rightarrow V$ of a neighborhood $U$ of $X$ in $X^{*}$ and put $\tilde{f}=r \circ f^{*}: U \rightarrow Y$. Observe that $d(\tilde{f} \mid X, f)<\varepsilon / 2$. For sufficiently large $i$, $j \geq i$ implies that $X_{j} \subseteq U$ so that $f_{j}=\tilde{f} \mid X_{j}$ is defined. By uniform continuity of $\tilde{f}$ on $X_{i}^{*}=X \cup \cup_{j \geq 1} X_{i}$ there is a $\delta>0$ such that $d\left(x, x^{\prime}\right)<\delta$ implies that $d\left(\tilde{f}(x), \tilde{f}\left(x^{\prime}\right)\right)<\varepsilon / 2\left(x, x^{\prime} \in X_{l}^{*}\right)$. Since for sufficiently large $j$ one has $d\left(p_{j}(x), x\right)<\delta$ for $x \in X$, one concludes that

$$
d\left(f(x), f_{J}^{\circ} p_{J}(x)\right) \leq d(f(x), \tilde{f}(x))+d\left(\tilde{f}(x), \tilde{f}\left(p_{j}(x)\right)\right)<\frac{\varepsilon}{2}+\frac{\varepsilon}{2}=\varepsilon
$$

for all $x \in X$.

(7.2) Definition. An inverse sequence $\underline{X}=\left\{X_{i}, p_{i t i+1}\right\}$ is called $e$-n-tame provided for every $i>0$ and every $\varepsilon>0$ there is a $j \geq i$, an at most $n$-dimensional compactum $K$, and maps $\alpha: X_{j} \rightarrow K$ and $\beta: K \rightarrow X_{i}$ with $\beta \circ \alpha \varepsilon$-close to $p_{i j}$.

Recall that a compactum $X$ approximately dominates a compactum $Y$ [Č2] if for every $\varepsilon>0$ there are maps $f: X \rightarrow Y$ and $g: Y \rightarrow X$ with $d\left(f \circ g, \mathrm{id}_{Y}\right)<\varepsilon$.

(7.3) Theorem. Let $\underline{X}=\left\{X_{i}, p_{t \imath+1}\right\}$ and $\underline{Y}=\left\{Y_{l}, q_{i t+1}\right\}$ be inverse $A A N R_{\mathrm{C}}$-sequences and let $\lim \underline{X}=\left(X, p_{\imath}\right)$ and $\lim \underline{Y}=\left(Y, q_{l}\right)$. If $\underline{X}$ is e-n-tame and $X$ approximately dominates $Y$, then $\underline{Y}$ is also e-n-tame.

Proof. Let an $i>0$ and an $\varepsilon>0$ be given. Since $X$ approximately dominates $Y$, there are maps $f: X \rightarrow Y$ and $g: Y \rightarrow X$ such that $d\left(f \circ g, \operatorname{id}_{Y}\right) \in \Lambda\left(q_{i}, \varepsilon / 6\right)$. Hence,

$$
d\left(q_{i} \circ f \circ g, q_{i}\right)<\varepsilon / 6 .
$$


By Lemma (7.1)(i), there is an index $i^{\prime} \geq i$ and a map $f_{i^{\prime}}: X_{i^{\prime}} \rightarrow Y_{i}$ with $d\left(f_{i^{\prime}} \circ p_{i^{\prime}}, q_{l} \circ f\right)<\varepsilon / 6$. Therefore,

$$
d\left(f_{i^{\prime}} \circ p_{i^{\prime}} \circ g, q_{i} \circ f \circ g\right)<\varepsilon / 6 .
$$

Combining (1) and (2), we get

$$
d\left(f_{i^{\prime}} \circ p_{i^{\prime}} \circ g, q_{i}\right)<\varepsilon / 3 .
$$

Now, since the inverse AANR $_{C}$-sequence $\underline{X}$ is $e$ - $n$-tame, there is an index $j^{\prime} \geq i^{\prime}$, an at most $n$-dimensional compactum $K$, and maps $\alpha^{\prime}: X_{j^{\prime}} \rightarrow K$ and $\beta^{\prime}: K \rightarrow X_{i^{\prime}}$ such that $d\left(\beta^{\prime} \circ \alpha^{\prime}, p_{i^{\prime} j^{\prime}}\right) \in \Lambda\left(f_{i^{\prime}}, \varepsilon / 3\right)$. Hence,

$$
d\left(f_{i^{\prime}} \circ \beta^{\prime} \circ \alpha^{\prime}, f_{i^{\prime}} \circ p_{i^{\prime} j^{\prime}}\right)<\varepsilon / 3 \text {. }
$$

Next, since $p_{j^{\prime}} \circ g: Y \rightarrow X_{j^{\prime}}$ is a map of $Y$ into an AANR $\mathrm{A}_{\mathrm{C}}$, by Lemma (7.1)(i), there is a $k \geq j^{\prime}$ and a map $g_{k}: Y_{k} \rightarrow X_{j^{\prime}}$ with $d\left(p_{j^{\prime}} \circ g, g_{k} \circ q_{k}\right) \in$ $\Lambda\left(f_{i^{\prime}} \circ p_{i^{\prime} j^{\prime}}, \varepsilon / 3\right)$. Thus,

$$
d\left(f_{i^{\prime}} \circ p_{i^{\prime}} \circ g, f_{i^{\prime}} \circ p_{i^{\prime} j^{\prime}} \circ g_{k} \circ q_{k}\right)<\varepsilon / 3
$$

because $p_{i^{\prime}}=p_{i^{\prime} j^{\prime}} \circ p_{j^{\prime}}$. From (3) and (5) we get

$$
d\left(f_{i^{\prime}} \circ p_{i^{\prime} j^{\prime}} \circ g_{k} \circ q_{k}, q_{i k} \circ q_{k}\right)<2 \varepsilon / 3,
$$

and from (4) we have

$$
d\left(f_{i^{\prime}} \circ \beta^{\prime} \circ \alpha^{\prime} \circ g_{k} \circ q_{k}, f_{i^{\prime}} \circ p_{i^{\prime} j^{\prime}} \circ g_{k} \circ q_{k}\right)<\varepsilon / 3 \text {. }
$$

If we apply Lemma (7.1)(ii) to (6) and (7), we see that there is an index $j \geq k$ so that

$$
d\left(f_{i^{\prime}} \circ p_{i^{\prime} j^{\prime}} \circ g_{k} \circ q_{k j}, q_{i j}\right)<2 \varepsilon / 3
$$

and

$$
d\left(f_{i^{\prime}} \circ \beta^{\prime} \circ \alpha^{\prime} \circ g_{k} \circ q_{k j}, f_{i^{\prime}} \circ p_{i^{\prime} j^{\prime}} \circ g_{k} \circ g_{k J}\right)<\varepsilon / 3 \text {. }
$$

Finally, (8) and (9) give us

$$
d\left(f_{i^{\prime}} \circ \beta^{\prime} \circ \alpha^{\prime} \circ g_{k} \circ q_{k j}, q_{i j}\right)<\varepsilon .
$$

Hence, if we put $\alpha=\alpha^{\prime} \circ g_{k} \circ q_{k j}: Y_{j} \rightarrow K$ and $\beta=f_{i^{\prime}} \circ \beta^{\prime}: K \rightarrow Y_{i}$, the last inequality can be rewritten as $d\left(\beta \circ \alpha, q_{i j}\right)<\varepsilon$, which proves that $\underline{Y}$ is $e$ - $n$-tame.

(7.4) Corollary. Let $\left(X, p_{i}\right)$ be the inverse limit of an inverse AANR ${ }_{\mathrm{C}}$-sequence $\underline{X}=\left\{X_{i}, p_{i+1}\right\}$. Then $\operatorname{dim} X \leq n$ iff $\underline{X}$ is e-n-tame.

Proof. Consider $X$ as a subset of the Hilbert cube $Q$. The corollary follows from the above theorem and Theorem (5.2) in [Č2], which says 
that $\operatorname{dim} X \leq n$ iff the inverse AANR $_{\mathrm{C}}$-sequence $\underline{N}=\left\{N_{l}, q_{i_{l}+1}\right\}$, where $N_{1} \supset N_{2} \supset \cdots$ is a decreasing sequence of compact ANR neighborhoods $N_{l}$ of $X$ in $Q$ with $X=\bigcap_{l>0} N_{l}$ and $q_{l i+1}: N_{i+1} \rightarrow N_{l}(j \geq i)$ are inclusions, is $e$ - $n$-tame.

\section{REFERENCES}

[Bo] S. A. Bogatyi, Approximate and fundamental retracts, (in Russian), Mat. Sbornik, 93 (135) (1974), 90-102.

[B] K. Borsuk, Theory of Shape, Monografie Matematyczne 59, Warsaw, 1975.

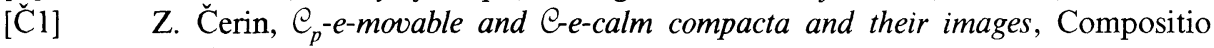
Math., 45 (1981), 115-141.

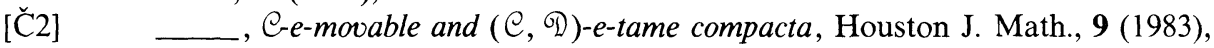
9-27.

[CD] D. Coram and P. F. Duvall, Approximate fibrations, Rocky Mountains J. Math., 7 (1977), 275-288.

[DM] K. Delinić and S. Mardešić, A necessary and sufficient condition for the n-dimensionality of invese limits, Proc. Internat. Sympos. on Topology and its Applications (Herceg-Novi, 1968), 124-129.

[FS] M. K. Fort, Jr. and J. Segal, Local connectedness of inverse limit spaces, Duke Math. J., 28 (1961), 253-260.

[Ge1] R. Geoghegan, Open problems in infinite-dimensional topology, Topology Proc., 4 (1979), 287-338.

[Ge2] __ Fibered stable compacta have finite homotopy type, Proc. Amer. Math. Soc., 71 (1978), 123-219.

[GM] G. R. Gordh, Jr. and S. Mardešić, Characterizing local connectedness in inverse limits, Pacific J. Math., 58 (1975), 411-417.

$[\mathrm{HH}] \mathrm{H}$. Hastings and A. Heller, Homotopy idempotents on finite-dimensional complexes split, Proc. Amer. Math. Soc., 85 (1982), 619-622.

[Hu] S. T. Hu, Theory of Retracts, Wayne State University Press, Detroit, 1965.

[KM] J. Krasinskiewicz and P. Minc, Generalized paths and pointed 1-movability, Fund. Math., 104 (1979), 141-153.

[M1] S. Mardešić, Strongly movable compacta and shape retracts, Proc. Internat. Sympos. on Topology and its Appl., (Budva 1972), Beograd 1973, pp. 163-166. 
[M2] _ Approximate polyhedra, resolutions of maps and shape fibrations, Fund. Math., 114 (1981), 53-78.

[MR] S. Mardešić and T. B. Rushing, Shape fibrations I, General Topology and its Appl., 9 (1978), 193-215.

[MSl] S. Mardešić and J. Segal, e-mappings onto polyhedra, Trans. Amer. Math. Soc., 109 (1963), 146-164.

[MS2] Movable compacta and ANR-systems, Bull. Acad. Polon. Sci., 18 (1970), 649-654.

[McR] L. F. McAuley and E. E. Robinson, On inverse convergence of sets, inverse limits, and homotopy regularity, Houston J. Math., 8 (1982), 369-388.

[No] H. Noguchi, A generalization of absolute neighborhood retracts, Kodai Math. Seminar Reports 1 (1953), 20-23.

[N] S. Nowak, Some properties of fundamental dimension, Fund. Math., 85 (1974), 211-227.

[Pa] B. Pasynkov, On the spectra and dimensionality of topological spaces, Math. Sbornik, 59 (99) (1962), 449-476.

[Sz] J. Szenthe, On the topological characterization of transitive Lie group actions, Acta Sci. Math., 36 (1974), 323-344.

[Wh] G. T. Whyburn, On sequences and limiting sets, Fund. Math., 25 (1935), 408-426.

Received April 12, 1982 and in revised form September 15, 1982. This paper was written during the author's visit to the Unviersity of Oklahoma while on leave from the University of Zagreb.

UNIVERSITY OF ZAGREB

41001 ZAGREB, PP. 187

YUGOSLAVIA

AND

MATEMATICKI INSTITUT

11000 BEOGRAD

YugoslaVia 


\section{PACIFIC JOURNAL OF MATHEMATICS \\ EDITORS}

DONALD BABBITT (Managing Editor)

University of California.

Los Angeles, CA 90024

Hugo Rossi

University of Utah

Salt Lake City, UT 84112

C. C. Moore and Arthur Ogus

University of California

Berkeley, CA 94720
J. DugundjI

Department of Mathematics

University of Southern California

Los Angeles, CA 90089-1113

R. FinN and H. Samelson

Stanford University

Stanford, CA 94305

\section{ASSOCIATE EDITORS}
R. ARENS
E. F. BECKENBACH
B. H. NeumanN
F. WOLF
K. YoshidA (1906-1982)

\section{SUPPORTING INSTITUTIONS}

UNIVERSITY OF ARIZONA

UNIVERSITY OF BRITISH COLUMBIA

CALIFORNIA INSTITUTE OF TECHNOLOGY

UNIVERSITY OF CALIFORNIA

MONTANA STATE UNIVERSITY

UNIVERSITY OF NEVADA. RENO

NEW MEXICO STATE UNIVERSITY

OREGON STATE UNIVERSITY
UNIVERSITY OF OREGON

UNIVERSITY OF SOUTHERN CALIFORNIA

STANFORD UNIVERSITY

UNIVERSITY OF HAWAII

UNIVERSITY OF TOKYO

UNIVERSITY OF UTAH

WASHINGTON STATE UNIVERSITY

UNIVERSITY OF WASHINGTON 


\section{Pacific Journal of Mathematics}

Vol. 112, No. $1 \quad$ January, 1984

Richard Blaine Barrar and Henry Loeb, Characterizing the divided difference weights for extended complete Tchebycheff systems $\ldots \ldots \ldots \ldots 1$

Harold Bennett and David John Lutzer, Generalized ordered spaces with

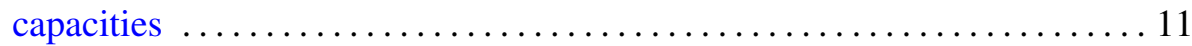

Geoffrey J. Butler and Lynn Harry Erbe, Comparison theorems for second-order operator-valued linear differential equations

Bohumil Cenkl and Richard D. Porter, de Rham theorem with cubical

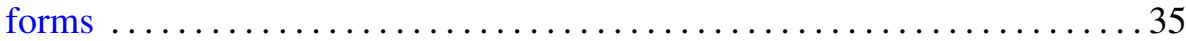

Zvonko Cerin, Characterizing global properties in inverse limits . ....... 49

Thomas Eugene Duchamp and Morris Kalka, Holomorphic foliations and deformations of the Hopf foliation .........................69 69

John Paul Hempel, Homology of coverings $\ldots \ldots \ldots \ldots \ldots \ldots \ldots \ldots \ldots$

Gerald Norman Hile and R. Z. Yeh, Inequalities for eigenvalues of the

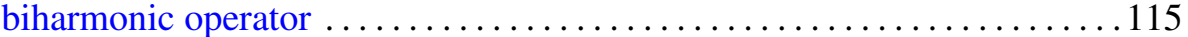

Kenneth Irwin Joy, A description of the topology on the dual space of a

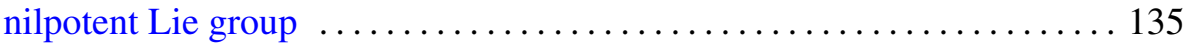

Alex Kumjian, On localizations and simple $C^{*}$-algebras $\ldots \ldots \ldots \ldots \ldots 141$

Bernardus de Pagter, The space of extended orthomorphisms in a Riesz

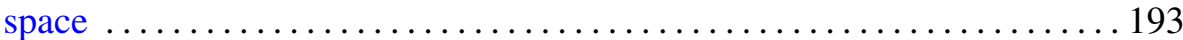

Stephen C. Persek, Iterated averaging for periodic systems with hidden

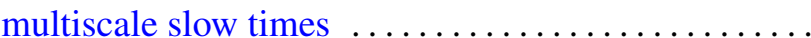

David Francis Rearick, Divisibility of arithmetic functions . . . . . . . . . 237

Masaaki Suzuki, The intrinsic metrics on the circular domains in $\mathbf{C}^{n}$ 